\title{
Attention Based Symmetry Detection in Colour Images
}

\author{
F. W. M. Stentiford \\ University College London \\ Adastral Park Campus, Martlesham Heath \\ Ipswich, UK \\ f.stentiford@ucl.ac.uk
}

\begin{abstract}
Symmetry is a fundamental structure that is found to some extent in all images. It is thought to be an important factor in the human visual system for obtaining understanding and extracting semantics from visual material. This paper describes a method of detecting axes of reflective symmetry in images that does not require prior assumptions about the image being analysed. The approach is derived from earlier work on visual attention that identifies salient regions and translational symmetries. Some results are reported on natural colour images taken from the Corel database.
\end{abstract}

Keywords - visual attention; symmetry; image retrieval

\section{INTRODUCTION}

Symmetries abound both in man made objects and in the structures to be found in nature itself. Symmetry is an important feature in natural scenes that attracts our attention and seems to guide the process of recognition. This has motivated many studies of symmetry and associated techniques that might be applied to image processing.

Symmetry analysis compares image regions and their transforms through translation, rotation and reflection in order to detect relevant structure. Most approaches avoid exhaustive search and reduce the enormous computational requirements by measuring intuitive features that characterise the presence of symmetrical structures. Marola [1] describes a method that can only be applied to shapes that are almost symmetric and requires the computation of the centre of mass. Sun et al [2] also make the assumption that the image is symmetric and measure the correlation between orientation histograms to detect planes of symmetry. Loy et al [3] use gradients to detect points of radial symmetry, but encounter problems of noise which are offset to some extent through the introduction of thresholds. Gradients and edges are also used by Reisfeld et al [4] who requires that symmetry transforms are local. Autocorrelation peaks are employed to determine the presence of symmetry in research by Liu et al. [5]. It was observed in this approach that significant parts of the image were overwhelmed by large expanses of background and that geometric distortions affected the results. Kiryati et al [6] develop a measure of local symmetry which is optimised using a probabilistic genetic algorithm.

\section{Visual Attention}

Salient regions in images may be detected through a process that compares small regions with others within the image [7]. A region that does not match most other regions in the image is very likely to be anomalous and will stand out as foreground material. For example, the edges of large objects and the whole of small objects normally attract high attention scores mainly because of colour adjacencies or textures that only occur rarely in the image. Repetitive backgrounds that display a translational symmetry are assigned low attention scores. No weight is given to the presence or otherwise of reflection or rotation symmetries.

Region matching requires a few pixels (a fork) within that region to match in a translated position in another region. If the difference in colour of one pixel pair exceeds a certain threshold a mismatch is counted and the attention score is incremented.

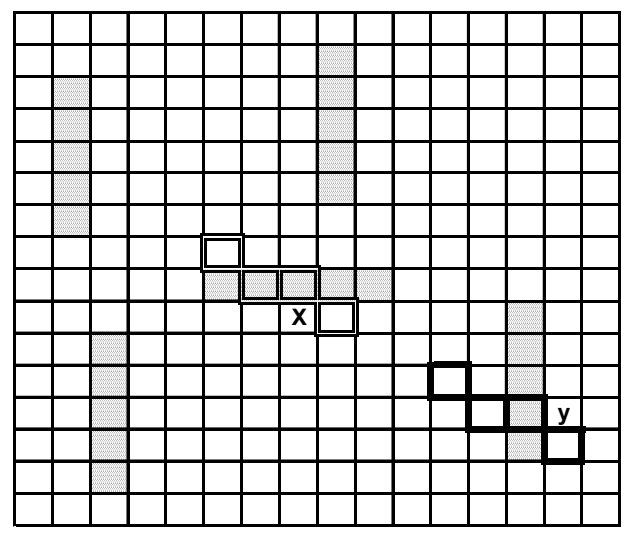

Figure 1. Fork at $\mathbf{x}$ mismatching at $\mathbf{y}$.

Let a set of measurements $\boldsymbol{a}$ correspond to a location $\boldsymbol{x}$ in bounded n-space $\left(x_{1}, x_{2}, x_{3}, \ldots, x_{n}\right)$ where

$$
\boldsymbol{x}=\left(x_{1}, x_{2}, x_{3}, \ldots, x_{n}\right) \text { and } \boldsymbol{a}=\left(a_{1}, a_{2}, a_{3}, \ldots, a_{p}\right)
$$

Define a function $\boldsymbol{F}$ such that $\boldsymbol{a}=\boldsymbol{F}(\boldsymbol{x})$

Consider a neighbourhood $\mathrm{N}$ of $\mathbf{x}$ where

$$
\left\{\boldsymbol{x}^{\prime} \in N \text { iff }\left|x_{i}-x_{i}^{\prime}\right|<\varepsilon_{i} \forall i\right\}
$$

Select a set of $\mathrm{m}$ random points $\mathrm{S}_{\mathbf{x}}$ in $\mathrm{N}$ (which we call a fork) where

$$
S_{\boldsymbol{x}}=\left\{\boldsymbol{x}_{1}^{\prime}, \boldsymbol{x}_{2}^{\prime}, \boldsymbol{x}_{3}^{\prime}, \ldots, \boldsymbol{x}_{m}^{\prime}\right\} \text { and } \boldsymbol{F}\left(\boldsymbol{x}_{i}^{\prime}\right) \text { is defined. }
$$



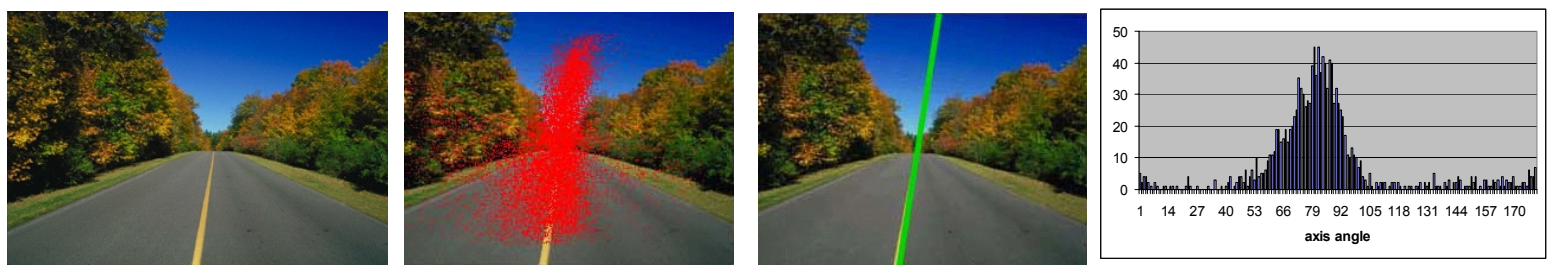

Figure 3. Original image, fork mid point distribution, reflection axis, and fork axis angle distribution

Select another location $\boldsymbol{y}$ for which $\boldsymbol{F}$ is defined.

Define the set $S_{\boldsymbol{y}}=\left\{\boldsymbol{y}_{1}^{\prime}, \boldsymbol{y}_{2}^{\prime}, \boldsymbol{y}_{3}^{\prime}, \ldots, \boldsymbol{y}_{m}^{\prime}\right\}$ where $\boldsymbol{x}-\boldsymbol{x}_{i}^{\prime}=\boldsymbol{y}-\boldsymbol{y}_{i}^{\prime}$ and $\boldsymbol{F}\left(\boldsymbol{y}_{i}^{\prime}\right)$ is defined.

The fork centred on $\mathbf{x}$ is said to match that of $\mathbf{y}$ if

$$
\left|F_{j}(\boldsymbol{x})-F_{j}(\boldsymbol{y})\right|<\delta_{j} \text { and }\left|F_{j}\left(\boldsymbol{x}_{i}^{\prime}\right)-F_{j}\left(\boldsymbol{y}_{j}^{\prime}\right)\right|<\delta_{j} \forall i, j .
$$

A location $\boldsymbol{x}$ will be worthy of attention if a sequence of $t$ forks matches only a few other neighbourhoods in the space. In the case of a two-dimensional still image (Fig. 1) a fork of $m=3$ pixels $x^{\prime}$ is selected in the neighbourhood of a pixel $x$. Each of the fork pixels might possess three colour intensities, so $\boldsymbol{F}(\boldsymbol{x})=\boldsymbol{a}=(r, g, b)$. The neighbourhood of a second pixel $\boldsymbol{y}$ matches the first if the colour intensities of all the corresponding pixels have values within $\delta$ of each other. The attention score $V(\boldsymbol{x})$ for each pixel $\boldsymbol{x}$ is incremented each time a mismatch occurs in the fork comparisons with a sequence of $\boldsymbol{y}$.

Pixels $\boldsymbol{x}$ that achieve high mismatching scores over a range of $t$ forks $S_{x}$ and pixels $\boldsymbol{y}$ are assigned a high estimate of visual attention. Applications to image compression and retrieval are described in [7], [8].

\section{Symmetry Detection}

In this paper symmetries are detected by transforming forks through reflections before translation and testing for match. Peaks in the distributions of reflection axis angles at which matches are found indicate the locations and strengths of the symmetries present in the image. Forks must include some $(h)$ pixels that mismatch each other otherwise large background tracts of self-matching sky, for example, would appear to exhibit trivial symmetries.

A fork of $\mathrm{m}$ random pixels $S_{x}$ is defined as a set of pixel positions where

$$
S_{x}=\left\{\boldsymbol{x}_{1}, \boldsymbol{x}_{2}, \boldsymbol{x}_{3}, \ldots, \boldsymbol{x}_{m}\right\} .
$$

A series of such forks is given by

$$
S_{x}^{k}=\left\{\boldsymbol{x}_{1 k}, \boldsymbol{x}_{2 k}, \boldsymbol{x}_{3 k}, \ldots, \boldsymbol{x}_{m k}\right\} \quad k=1,2, \ldots, M
$$

with $\left|F_{j}\left(\boldsymbol{x}_{p k}\right)-F_{j}\left(\boldsymbol{x}_{q k}\right)\right|>\delta_{j}$ for at least $h$ values of $p$.

A series of randomly translated and reflected forks of $\mathrm{m}$ pixels $S_{y}^{k}$ is defined where

$$
\begin{gathered}
S_{y}^{k}=\left\{\boldsymbol{y}_{l k}, \boldsymbol{y}_{2 k}, \boldsymbol{y}_{3 k}, \ldots, \boldsymbol{y}_{i k}\right\} \\
\text { and } \boldsymbol{y}_{i k}-\boldsymbol{y}_{1 k}=R_{\theta}\left[x_{i k}-x_{1 k}\right]^{\prime} \forall i, k \\
\text { and } R_{\theta}=\left[\begin{array}{cc}
\cos 2 \theta & \sin 2 \theta \\
\sin 2 \theta & -\cos 2 \theta
\end{array}\right], \alpha_{1} \leq \theta \leq \alpha_{2}
\end{gathered}
$$

where $\alpha_{1}$ and $\alpha_{2}$ are lower and upper bounds on the choice of $\theta$.

The fork $S_{y}^{k}$ matches indicating a symmetry if

$$
\left|F_{j}\left(\boldsymbol{x}_{i k}\right)-F_{j}\left(\boldsymbol{y}_{i k}\right)\right|<\delta_{j} \forall i, j .
$$

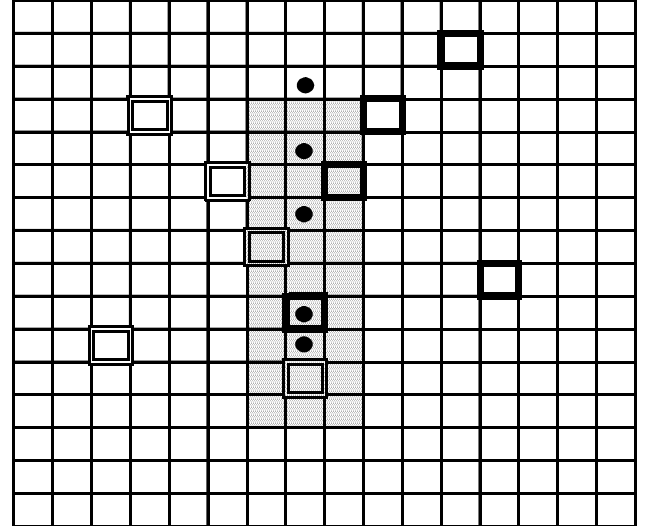

Figure 2. Vertically symmetric forks

Fig. 2 shows a vertically symmetric pair of 5 pixel forks matching a vertically symmetric shape. The mid points of lines joining corresponding fork pixels will tend to lie along the axis of symmetry of the shape as indicated by the dots.

Distributions of reflection or rotation symmetries are computed in the following steps:

1. Set histogram to zero.

2. Generate $S_{x}^{k}$ with $\left|F_{j}\left(\boldsymbol{x}_{p k}\right)-F_{j}\left(\boldsymbol{x}_{q k}\right)\right|>\delta_{j}$ for at least $h$ values of $p$

3. Generate $S_{y}^{k}$ at random $\theta, y$

4. If no match is found loop to step 3, $P$ times else increment histogram at $\theta$ following a match.

Loop to step $2, k=M$ times.

\section{Results}

Parameter values used to generate forks and symmetry distributions reported here are $\mathrm{m}=12, \mathrm{~h}=3, \mathrm{M}=10000, \mathrm{P}=$ $100, \alpha_{1}=0^{\circ}, \alpha_{2}=180^{\circ}, \delta=80$ where r,g,b values range from $0-255$. The location of axes of reflection can be revealed by plotting pixels at the mid points of corresponding pixels in matching forks. The mid points will lie on the axis that was used to generate the reflected fork and a concentration of plotted points will indicate the presence of an axis of reflective symmetry. Fig. 3 shows a colour image (320x214) selected from the Corel database together with a display of the mid points in matching forks, a display of the optimum axis of reflection, and the distribution of reflection axis angles for matching forks. The distributions of mid points and axis angles indicates a range of axes of symmetry from $50^{\circ}$ to $100^{\circ}$ with the main peak at $81^{\circ}$. 
Fig. 4 shows another scene with the axis angle distribution peaking at $90^{\circ}$. A less significant peak near $180^{\circ}$ and the mid point distribution indicate some symmetry also across a horizontal axis. Fig. 5 has an axis tilted to the right at $88^{\circ}$ possibly because of the asymmetric vegetation on the gateway. The image in Fig. 6 gives rise to a greater spread of axis angles with a peak tilted to the left at $91^{\circ}$ and a greater presence of horizontal symmetries. The lower contrast in colour/brightness distribution may have contributed towards a higher probability of fork matching at all axis angles. The smoothing that is applied to the axis angle distributions yields a near vertical $\left(91^{\circ}\right)$ dominant maximum in Fig. 7, but the colonnade of trees allows a range of axis angles with the horizontal again producing a subsidiary peak. In the case of Fig. $8 \delta$ was reduced to a value of 50 to encourage more colour discrimination in the foliage and in turn a sharper indication of the axis of symmetry at $90^{\circ}$. Again a measure of horizontal symmetry is seen. Finally the strong axis of vertical symmetry is identified along the line of the car bonnet in Fig. 9.

\section{Discussion}

The results presented here are derived using the basic RGB colour space with very broad thresholds $\delta_{j}$ to distinguish colours. Some preliminary experiments indicate that other colour spaces such as Lab, HSV, etc do not affect the results in a significant way. However, further optimisation of the threshold values on a per image basis should yield more informative results.

It should be noted that reflection axis distributions reflect the average of the whole image. It is reasonable to assume that specific regions of the image may have localised symmetrical structure that would not be revealed in such a global measure. For example the symmetry apparent in road markings may be obscured by more pervasive image structure.

The approach to identifying symmetry is based on a trial and error process of generating features (forks) that are present in transformed pairs. No guidance is given during this process as this would inevitably damage the performance on certain classes of images by precluding relevant features from being produced. The negative effects of such guidance cannot be predicted in advance of experiment and would not be discovered until sufficient data is explored.

The results reported in this paper have been produced with 10000 iterations of fork generation in order to yield accuracies of the order of one degree. Although the computational steps are very simple there are a large number of them and a symmetry analysis takes about 10 seconds on a $1.8 \mathrm{GHz}$ machine running in $\mathrm{C}++$. However, the matching of forks can be carried out in parallel as each match is independent of the next and related implementations on the Texas Instruments DM642 DSP platform indicate that processing can take place at video speeds.

\section{Conclusions}

This paper has described a technique for extracting symmetries from natural colour images that does not require manual intervention or the prior specification of features that characterise those symmetries. The features or forks are produced through a modified attention focussing mechanism that selects the best combination of positional and reflection transforms that maximises the matching of forks. Despite much asymmetry that is present in all the images the principal axes of reflective symmetry are extracted.

Future work will explore other fork transforms that should reveal other symmetries such as rotation and perspective in natural images. This will be used to derive metadata describing image relationships that can be used in Content Based Image Retrieval applications.

\section{ACKNOWLEDGMent}

This research has been conducted with the support of BT and within the framework of the European Commission funded Network of Excellence "Multimedia Understanding through Semantics, Computation and Learning" (MUSCLE).

\section{REFERENCES}

[1] G. Marola, "On the detection of the axes of symmetry of symmetric and almost symmetric planar images," IEEE Trans on PAMI, vol 11, pp 104-108, 1989.

[2] C. Sun and J Sherrah, "3D symmetry detection using the extended Gaussian image,” IEEE Trans on PAMI, vol 19, no 2, pp 164-168, 1997.

[3] G. Loy and A. Zelinsky, "Fast radial symmetry for detecting points of interest," Trans on PAMI, vol 25, no 8, pp 959-973.

[4] D Reisfeld and H. W. Y. Yeshurun, "Context free attentional operators: the generalised symmetry transform," Int. J. Comp. Vis., vol 14, pp 119-130, 1995.

[5] Y. Liu and R. T. Collins, "A computational model for periodic pattern perception based on frieze and wallpaper groups," Trans on PAMI, vol 26, no 3, March 2004.

[6] N. Kiryati and Y. Gofman, "Detecting symmetry in grey level images: the global optimization approach," Int. J. Comp. Vis., vol 29, pp29-45, 1998.

[7] F. W. M. Stentiford, "Automatic identification of regions of interest with application to the quantification of DNA damage in cells," Proc. SPIE Vol. 4662, pp 244-253, 2002.

[8] F. W. M. Stentiford, "An estimator for visual attention through competitive novelty with application to image compression", Picture Coding Symposium, Seoul, 2001.

[9] Multimedia Understanding through Semantics, Computation and Learning, Network of Excellence, EC $6^{\text {th }}$ Framework Programme, FP6-507752, http://www.muscle-noe.org/general.html 

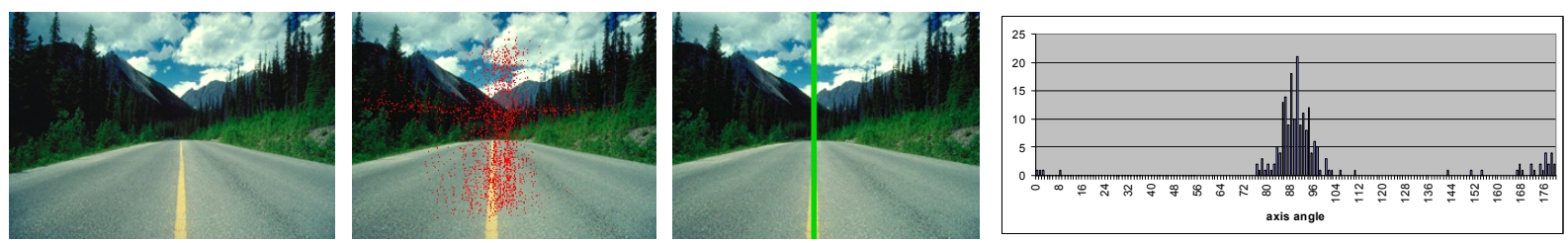

Figure 2. Original image, fork mid point distribution, reflection axis, and fork axis angle distribution
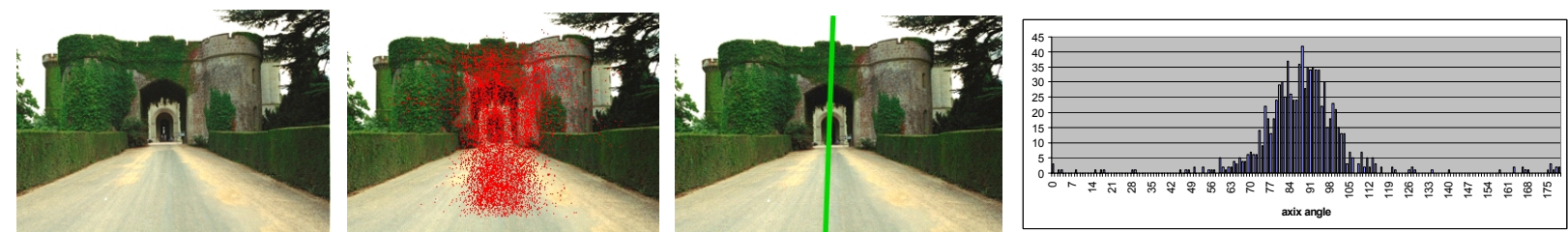

Figure 3. Original image, fork mid point distribution, reflection axis, and fork axis angle distribution
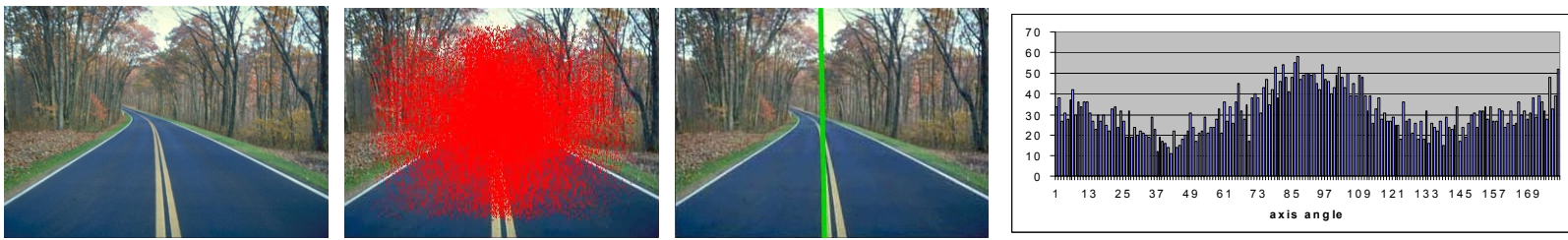

Figure 4. Original image, fork mid point distribution, reflection axis, and fork axis angle distribution
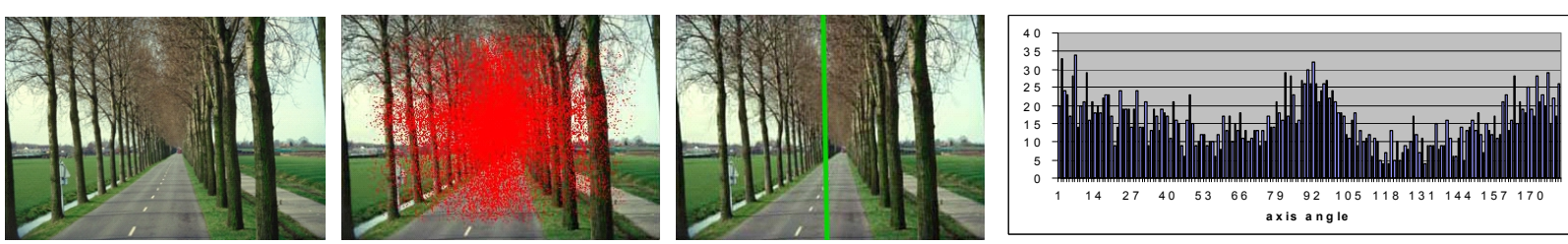

Figure 5. Original image, fork mid point distribution, reflection axis, and fork axis angle distribution
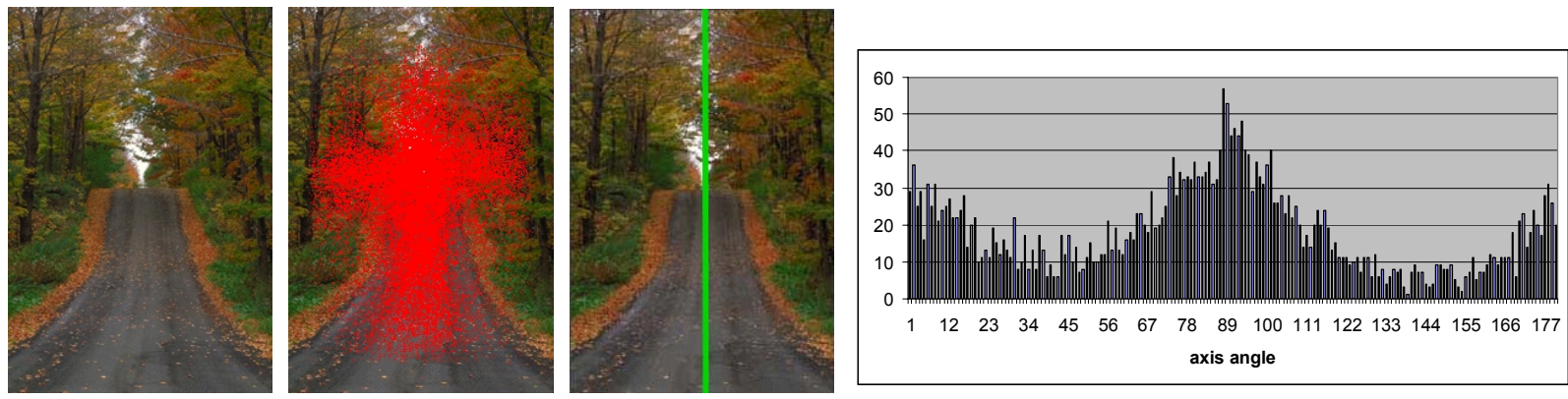

Figure 6. Original image, fork mid point distribution, reflection axis, and fork axis angle distribution
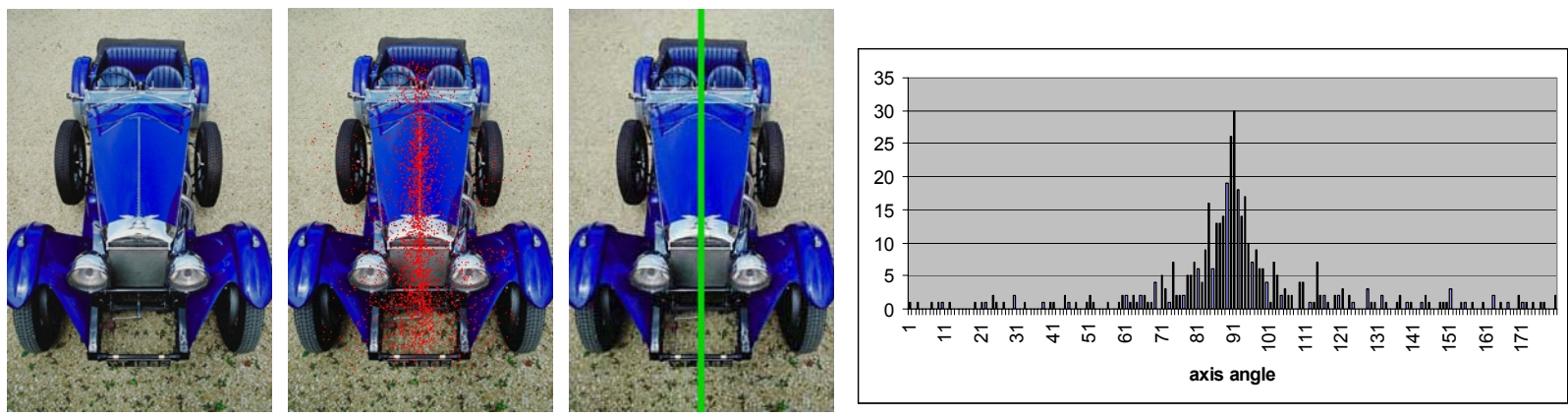

Figure 7. Original image, fork mid point distribution, reflection axis, and fork axis angle distribution 